\title{
Can shape be perceived by dynamic touch?
}

\author{
GREGORY BURTON and M. T. TURVEY \\ Center for the Ecological Study of Perception and Action \\ University of Connecticut, Storrs, Connecticut \\ and \\ H. YOSEF SOLOMON \\ University of Cincinnati, Cincinnati, Ohio
}

\begin{abstract}
The possibility that some aspects of the shapes of solid objects can be perceived through dynamic touch, even when the objects are not touched, but simply wielded with a handle, was investigated in four experiments. Wooden solids were constructed of three sizes and five shapes: hemisphere, cylinder, parallelepiped, cone, and pyramid. Experiments 1 and 2 involved comparisons (judgments of same or different) between and among wielded objects of the same mass. In Experiments 3 and 4, subjects were required to wield an object and to select a match from a visible arrangement of objects of the five shapes; the wielded objects were of two sizes, each different from that of the visible objects. The success of subjects at these tasks, and the patternings of errors, are seen to involve the characteristic moment of inertia profiles of each shape, and a ratio of the object's resistances to rotation around orthogonal axes is shown to be a strong predictor of performance in the identification experiments. The results are discussed with reference to dynamic touch and to the notion of shape invariants that do not reduce to aspects of object surface.
\end{abstract}

Existing research on nonvisual perception of object shape (e.g., J. J. Gibson, 1963; Jenkins, 1947; Klatzky, Lederman, \& Metzger, 1985; Lederman \& Klatzky, 1987; Roland \& Mortensen, 1987; Streri \& Pêcheux, 1986) has tended to focus on the perceiving that accompanies the feeling or enclosing of an object with one or both hands. For vision, the shape of an object is the particular arrangement or layout of its surface or surfaces. Similarly, for touch, where one or both hands explore an object, shape is defined by surface arrangement. It is the case, however, that there are aspects of an object's material layout that are not exclusively accessible to contact (visual or tactual) with the object's surface-specifically, the moments of its mass distribution, $\Sigma m \mathbf{r}^{\circ}, \Sigma m \mathbf{r}^{1}, \Sigma m \mathbf{r}^{2}$, defining, respectively, mass, static moment, and moment of inertia (where $\mathbf{r}$ is the position vector of a small element of mass $m$ ). These moments have implications for the utility that the object offers to the perceiver, and do not necessarily imply the encompassing of the object's surface or a sampling of its criterial vertices, edges, or faces, for their registration. For example, the moments could be registered by wielding the object about a fixed point.

This research was made possible by a grant from the National Science Foundation (BNS-8720144). The authors would like to acknowledge the contributions of Cliff Ward and colleagues at the University Carpentry Shop, for construction of the solids, Andrew Brenc and Kevin Parzych for assistance in running the subjects, Claudia Carello for preparation of some of the figures, and Myron Braunstein, Bradley Carlin, Ronald Growney, James Green, Leonard Katz, and Uwe Koehn for statistical advice. M. T. Turvey is also at Haskins Laboratories. Reprint requests should be sent to $M$. T. Turvey, Center for the Ecological Study of Perception and Action, Department of Psychology, University of Connecticut, Storrs, CT 06269-1020.
J. J. Gibson (1966) proposed a division of touch into a number of major perceptual subsystems, including cutaneous touch, haptic touch, and dynamic touch (see also Loomis \& Lederman, 1986). Cutaneous touch involves pressure on the skin and deeper tissues without movement of the joints; haptic touch involves stimulation of the skin and deeper tissues with movements of the joints; and dynamic touch involves stimulation of the skin and joints in combination with muscular exertion. Although muscular exertion is involved in feeling the arrangement of an object's surface or surfaces, dynamic touch is not implicated, because the muscular exertion is not intrinsic to the possibility of perception. When an object is wielded with an unchanging relation between the hand and the object, muscular exertion is crucial to the perceiving, and the touching is, unequivocally, dynamic or effortful.

In this article, we pursue the idea that because the shape of a solid object has mechanical implications, an approximation to ordinary shape perception might be achievable through dynamic touch in the absence of the viewing or feeling of a surface. ${ }^{1}$ (We might compare this claim to the celebrated analysis of Kac [1966; see also Strang, 1986], who considered whether the shape of a drum might have a perceptible influence on the sound produced when it is struck.) This conjecture grows out of previous work that demonstrates the contribution of an object's moment of inertia to the perception of the object's length (Solomon \& Turvey, 1988; Solomon, Turvey, \& Burton, 1989a, $1989 \mathrm{~b}$ ), and object orientation with respect to the hand (Turvey, Solomon, \& Burton, 1989), with both perceptions tied to the resistances of the objects to rotational acceleration. 
Objects of different shapes differ in the resistances they offer to being rotated in different directions. For example, a long, thin object with negligible diameter may offer considerable resistance to being whipped up and down, or side to side, but may exhibit little resistance to being twisted (rotated about its long axis). In contrast, a wide flat object, such as a disk with a handle perpendicular to its center, may show little resistance to being rotated up and down but will offer more resistance to being rotated about the axis through the handle.

Speaking generally, any given object has a particular pattern of resistances to rotation with respect to a fixed point of rotation, summarized by the inertia tensor I (e.g., Goldstein, 1980; Kibble, 1985). The tensor can be represented by a $3 \times 3$ matrix. The terms on the diagonal, $I_{x x}, I_{y y}$, and $I_{z z}$, stand for the object's resistance to rotation about each of the three axes through the arbitrary origin (Figure 1). Thus, the long rod in the example above may have large values of $I_{x x}$ (whipped side to side) and $I_{y y}$ (whipped up and down), but a low value of $I_{z z}$ (twisted). The off-diagonal terms of I represent the object's resistances to rotation in directions perpendicular to the rotations about the axes; they are termed the products of inertia. For an object rotating about one of its central principal axes (those that pass through the center of mass), the products of inertia are zero, meaning that there is no resultant force or couple on the axis. The central principal axes are fixed in the object, not in space, and rotate with it. Differently shaped objects made of the same material and conforming to the same density function (mass per unit volume equals a constant, and all elemental volumes are occupied; that is, there are no holes), are objects that are distinguished systematically by their principal moments of inertia, those about their principal axes. Under these constraints, each object's shape at its given size would be formally associated with a particular triad of principal moments, and each object's shape at any size would be formally associated with a relation among the principal moments that was constant over

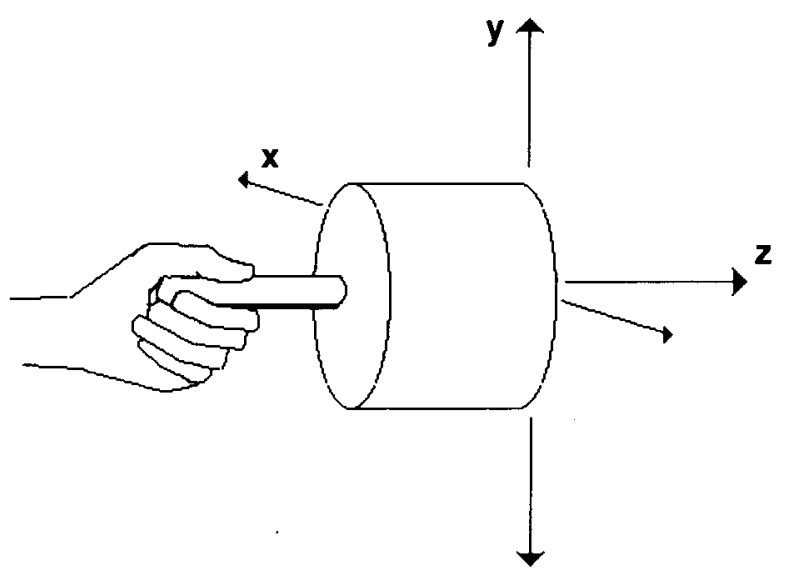

Figure 1. Depiction of the three axes about which the resistances to rotational acceleration of $\mathbf{a}$ hand-held object are defined. size variation. Given the demonstrated sensitivity of dynamic touch to rotational moments, the question arises, therefore, of whether or not the sensitivity extends to an invariant relation among the invariants of rigid body motion, the principal moments. If it does, an approximation to ordinary shape perception, which is tied to surface arrangement and typically achieved through looking or feeling with the hands, would seem to be achievable through dynamic touch (for example, by wielding).

A major barrier to this possibility of a kind of shape perception that occurs through dynamic touch can be readily identified, however. Whereas a given object of a given shape maps to one diagonalized inertia tensor, the inverse mapping is one to many. That is, given three principal moments of inertia, there are indefinitely many shapes to which they could apply. The implication, therefore, is that even if the principal moments were extractable by means of effortful touch, there would be no guarantee that a person would thereby be aware of any particular shape. This issue of the physical equivocality of the inverse mapping, and a potential ambiguity of perception, arises in the simpler case of perceiving extent by wielding. Significantly, a consideration of this simpler case will suggest that we can expect to see shape-specific responses despite equivocality in the principal moments-to-shape mapping.

A rod of given density and length possesses a definite resistance to rotation about an end. The mapping of rod magnitudes to moment of inertia is one to one. In sharp contrast, the mapping from a given moment of inertia to rod magnitudes is many to one. Thus, a rod of mass $50 \mathrm{~g}$ and length $50 \mathrm{~cm}$ has a moment of inertia about an end of $41,666.7 \mathrm{~g} / \mathrm{cm}^{2}$. Given a moment of inertia of $41,666.7 \mathrm{~g} / \mathrm{cm}^{2}$, one could suppose that the object in question was $2 \mathrm{~g} / 250 \mathrm{~cm}, 150 \mathrm{~g} / 28.9 \mathrm{~cm}, 10 \mathrm{~g} / 111.8 \mathrm{~cm}$, or $500 \mathrm{~g} / 15.8 \mathrm{~cm}$, and so on. Even if the mass of the object were given, the indeterminacy would remain with regard to length. Moment of inertia divided by mass is $K^{2}$, the squared radius of gyration. $K$ is a complex quantity. Besides the characteristic length dimensions of the object (that could include width or radius in addition to length), it involves a constant of proportionality that varies with object shape and the distance of the rotation point from the center of mass. In light of these mathematical indeterminacies, two facts should be noted. First, perceived reachable distance with a wielded rod is a single-valued function of moment of inertia (Solomon \& Turvey, 1988; Solomon et al., 1989a, 1989b). That is, people feel a given rotational inertia as a single well-defined extent, and not as multiple possible extents. Second, when the rods are of uniform density throughout their lengths (no additional masses have been affixed to the rods), perceived reachable distances are always in the ballpark of the actual reachable distances and sometimes match them identically. For example, given steel rods of $30.5,45.7,61.0$, 76.2 , and $91.4 \mathrm{~cm}$ held at their proximal ends, mean perceived reachable distances were $31.9,46.4,65.2,80.9$, and $92.4 \mathrm{~cm}$ (Solomon \& Turvey, 1988, Experiment 2). 
The implications of perceiving rod length by wielding for perceiving object shape by wielding are two: First, hidden constraints are present in the haptic system's exploitation of rotational inertia, such that single-valued perceptual functions are obtainable in the face of mathematical indeterminacy; second-and related-given a particular pattern of principal moments of inertia, a particular shape perception could result. Four experiments were designed to test the latter expectation.

\section{EXPERIMENT 1}

The preeminent goal in these experiments was to determine the extent to which observers can identify the shape of a solid object that is wielded but not viewed or touched. This ability was expected to depend on the detection of pervasive, intrinsic mechanical properties of objects of different shapes, in contrast to the surface properties that are perceivable to different extents when observers view or manually explore the objects in question. It is also worth noting that the criteria for categorizing objects of different shapes are also conventionally framed in the language of surface properties, or with respect to the number and relationship of faces and vertices. Mechanical aspects of shape may not show the same form, and consequently, the criteria by which objects of different shapes will be most distinctive under dynamic touch are very much open to question.

The shapes chosen for presentation in each of the following experiments (hemisphere, parallelepiped, cylinder, cone, quadrilateral pyramid) form a set that is quite varied from a conventional perspective, and each of the shapes can be easily defined in terms of faces and vertices. Our eventual goal was to determine whether subjects could use the same names to identify solids that they wielded, but it is also important to learn if shapes that are distinguishable visually are equally distinguishable haptically; that is, are comparisons that are easy to make visually also easy when the solids are wielded? This question requires a methodology that involves only the comparison of shapes, as opposed to individual identification. Experiment 1 was designed to test subjects' abilities to make a fairly rudimentary decision about two unseen, untouched solids-whether their shapes were the same or different.

\section{Method}

Subjects. Eight graduate students associated with the University of Connecticut participated on a volunteer basis. Four subjects were male and 4 female; all 8 subjects were right-handed. Most of the subjects had some experience with haptic experiments.

Materials. Ten wooden solids were constructed of pine, representing two each of five shapes (hemisphere, cylinder, parallelepiped, cone, and pyramid). Three sets of these solids were built; the smallest was employed in Experiment 1. The dimensions of the solids are given in the first column of Table 1 . These dimensions were chosen so that the volumes of the objects (and consequently, the mass) would be as close to equal as was practicable. The mass of each solid (including handle), determined empirically by weighing, is given in the first column of Table 2; it is clear from Table 2 that the solids were not totally equivalent in mass, reflecting the
Table 1

Dimensions (in Centimeters) of the Three Sizes of Solids Used in the Experiments

\begin{tabular}{cccc}
\hline Shape & Size 1 & Size 2 & Size 3 \\
\hline $\begin{array}{l}\text { Hemisphere } \\
\text { Height }\end{array}$ & 8.38 & 9.65 & 10.69 \\
Radius & 8.38 & 9.65 & 10.69 \\
Cylinder & & & \\
Height & 9.91 & 12.19 & 13.72 \\
Radius & 6.35 & 6.99 & 7.62 \\
Parallelepiped & & & \\
$\quad$ Height & 9.65 & 11.68 & 12.70 \\
Width & 11.43 & 12.70 & 13.97 \\
Cone & & & \\
Height & 29.46 & 36.58 & 41.15 \\
Radius & 6.35 & 6.99 & 7.62 \\
Pyramid & & & \\
Height & 28.70 & 34.80 & 38.35 \\
Width & 11.43 & 12.70 & 13.97 \\
\hline
\end{tabular}

Table 2

Mass (in Grams) of the Solids Used in the Experiments

\begin{tabular}{lllr}
\hline \multicolumn{1}{c}{ Shape } & Size 1 & Size 2 & Size 3 \\
\hline Hemisphere & 486.0 & 604.0 & $1,002.5$ \\
Cylinder & 521.0 & 722.0 & 939.0 \\
Parallelepiped & 525.0 & 750.0 & $1,008.5$ \\
Cone & 525.0 & 964.0 & $1,138.0$ \\
Pyramid & 525.5 & 746.5 & $1,022.5$ \\
\hline
\end{tabular}

difficulty of planing such objects to precise standards and with precisely equivalent raw materials. Consequently, the mass differences of the experimental objects will be tested as alternate influences on observer judgments of the objects. Uniform handles (length, $12.7 \mathrm{~cm}$; radius, $.95 \mathrm{~cm}$ ) were inserted in the center of the base of each object, as depicted in Figure 1.

Apparatus. The experiment was conducted in a large classroom that also contained equipment not related to the present study. The subjects sat in an armless chair.

Procedure. While blindfolded, the subject was given 2 of the 10 objects, which were held only by the handle and at the same place on the handle. The subject was instructed to shake the shapes in any way desired (as long as they were held by the handle) and report whether the 2 objects were same or different in shape. The subject was given a demonstration beforehand of some of the styles of shaking that could be informative and was warned not to strike the objects against one another, the chair, or the parts of the body. There was no time limit to the exploration.

Each subject was given nine practice trials with the solid objects. In the first five, each subject was given a pair of objects of the same shape; the subject knew they would be the same and was instructed to try to feel the similarity of the objects. All five shapes were used. In addition, the subject was invited to try to determine the actual shape of the two objects, although the subject knew that this would not be required in the main task. For the next two practices, the subject was given a different pair for comparison, followed by another same; as before, the subject was told whether the shapes were the same or different. Finally, there were two more practices in the style of the main trials, in which the subject was asked to judge whether the shapes held were the same or different.

For the last 6 subjects, a switch option was added. The subject could request for the objects to be exchanged, so that the object formerly in the left hand was afterward in the right, and vice versa. This option was included because subjects reliably reported that 
the same object felt different according to the hand in which it was held.

For each subject, each shape was paired with itself four times and with each of the other shapes twice, making 40 trials, of which an equal number involved same comparisons and different comparisons. (Due to an error in presentation, 1 subject was given one extra same comparison and one fewer different comparison. This is reflected in the totals in Figure 2.) In comparisons of different shapes, each shape was once held in the left hand and once held in the right. The 40 trials were randomized differently for each subject.

The subjects were permitted to view all of the solids before any trials were conducted.

\section{Results and Discussion}

The results of Experiment 1 are given in Figure 2. It can be seen that different shape combinations were more or less likely to be confused-for example, no subject ever reported "same" when the objects presented were a cone and a hemisphere, whereas a cylinder and a parallelepiped were judged (incorrectly) to be the same more often than they were judged (correctly) to be different. The mean number of hits (same objects judged as same) was 10.44 (out of a maximum of 20); the mean number of false alarms (different objects judged as same) was 4.55 (these values for the subject who was presented with an extra same trial were normalized according to the actual number of same and different trials presented). A $t$ test conducted on these values indicated a significant difference between hits and false alarms $[t(7)=4.36, p<.01]$; note that this analysis takes into account the fact that par-

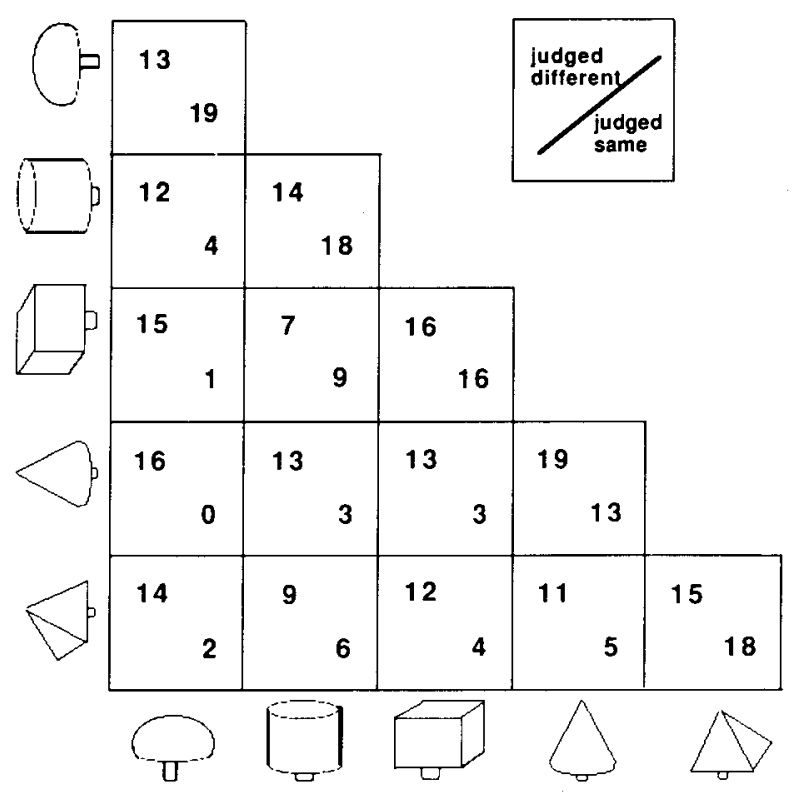

Figure 2. Results for the 8 subjects in Experiment 1. The value in the upper left of each figure represents the number of times the two objects were judged to be different; the value in the lower right indicates the number of times the combination was judged to be the same. Thus, if the shape along the top is the same as the shape along the side, the upper number designates the number of incorrect responses; the pattern is reversed if the two shapes were different. ticular subjects may be biased toward reporting "same" or "different." 2

The results of Experiment 1 indicate that (1) solid objects of different shapes are distinguishable by means of wielding, and (2) there is a tendency to judge the solids as different even when they are the same. In the experiment, judgments were arrived at by wielding two objects simultaneously, one in each hand. Differences between the two hands with respect to sensitivity to inertial properties has been noted. Brodie (1988) reports that, in some experimental conditions, perceived heaviness of an object varies systematically with the hand that does the lifting. Sensitivity to inertial qualities may vary in subtle ways with the parameters of the exploratory activity - the manner in which the objects are hefted or wielded. If so, then Guiard's (1987) argument that the two hands operate on different spatial and temporal scales in bimanual motor tasks could have some bearing on Brodie's (1988) observation, and on that summarized in point (2) above.

\section{EXPERIMENT 2}

Given the outcome of Experiment 1, it seems that a more effective paradigm would be one in which subjects judge the relative (rather than absolute) similarity of shapes in the different hands. In Experiment 2, subjects were given three wooden solids of closely similar mass, two of which were the same and one of which was different in shape. Their task was to select which two of the objects were most similar in shape. Half of the objects used in Experiment 2 were the same as those in Experiment 1; the other objects differed only in that solids of a larger size were employed, in order to preclude the possibility that the abilities of the subjects depended on a particular object set.

\section{Method}

Subjects. Sixteen undergraduates at the University of Connecticut participated for partial fulfillment of course requirements. Thirteen females and 3 males participated; 3 of the women and 1 of the men were left-handed. None of the subjects had participated in Experiment 1.

Materials. In the experiment, an apparatus was employed in which the shapes could stand upright. This device or stand was composed of a metal rail on which three metal fixtures were attached; these fixtures are customarily used to attach rails in order to make a raised floor, but the slot in the fixtures was large enough to fit the handles of the shapes and tight enough so that the shapes would stand vertically. The subjects could easily take the shapes from the fixtures by grasping their handles. The stand was mounted on a wooden board, which was tightly clamped to a table.

In Experiment 2, we used two objects in each of the five shapes, in the two lowest sizes identified in Tables 1 and 2 . The geometrical dimensions of the second set were also constrained to be similar to those in the smaller set; as is evident from Table 1, the correspondence is close, but not exact. The handles for the medium set were identical to those used in the small set.

Apparatus. The same equipment was used as in Experiment 1; the experiment was conducted in a smaller room.

Procedure. The subject's task in Experiment 2 was to explore the three solids that were placed in the stand on each trial and report which of the three was different in shape from the other two. The 
subjects were allowed to pick up the objects in any order and to shake them in the same ways as had been allowed in Experiment 1. The subjects were also permitted to return to any or all of the three shapes as often as desired. The subjects were able to take the shapes from the fixtures easily, but for returning the shapes, they were allowed simply to hold the shape over the desired fixture, at which point the experimenter would replace the object.

Each object was paired with every other object twice, once with two of the first and one of the second, and once with two of the second and one of the first (e.g., there was both a trial with two pyramids and a cone, and one with two cones and a pyramid). This yielded a total of 20 trials for each size ( 10 possible combinations $\times 2$ orders); 8 of the subjects were presented with the medium objects and 8 were presented with the small objects. The different object in each trial was randomly assigned to the leftmost, middle, or rightmost fixture.

In contrast to the procedure in Experiment 1, the subjects were given no practice trials, and neither viewed any of the solids beforehand nor were told how many different shapes might be presented.

\section{Results and Discussion}

The left panel of Figure 3 reveals the results for the Size 1 objects; the corresponding results for the Size 2 objects are given in the right panel. Overall performance was significantly better than chance $[t(7)=11.02$, $p<.001$ ], where the average number of combinations in which the subject correctly picked the different shape (13.7 out of 20) was compared to the chance level of 6.7 (i.e., 1 out of 3 trials). Analysis of variance revealed that the number of correct judgments was significantly different for the two sizes $[F(1,14)=5.52, p<.05]$, with the Size 2 objects more easily discriminated. An ANOVA also confirmed that the various shape combinations differed in the accuracy shown by subjects $[F(9,126)=5.98$, $p<.001$ ]. It can be seen from Figure 3 that combinations of cylinders and cylinders and pyramids were particularly difficult to judge, whereas a combination such as hemisphere, hemisphere, and cone or hemisphere, hemisphere, and pyramid was almost never judged incor- rectly. The interaction between object size and combination was not significant.

The results indicate that subjects can distinguish some shapes by wielding them, even when the subjects have no foreknowledge of the kinds of object shapes that they are wielding (recall that in Experiment 2, unlike Experiment 1 , the subjects were neither told about the objects nor given the opportunity to view them). The results also suggest that the criteria that make viewed objects distinctive in shape may not be as salient under dynamic touch. An alternate supposition is that the wielding of the objects in these experiments does not depend on different criteria, but instead reflects a reduced sensitivity to the same criteria. For example, we could suppose that a cylinder and a parallelepiped might look more alike than, say, a hemisphere and a cone; in fact, they might be visually indistinguishable under a filter that reduces highfrequency edge information (just to name one possibility). The supposition that different criteria are employed is more plausible physically (in that it is not clear how aspects of faces and vertices, in contrast to mechanical properties like those in the inertia tensor, affect the tissue strains and rates of strain at the hand or wrist when an object is wielded), but the contribution of mechanical properties was not demonstrated conclusively by the results of the first two experiments. Such a demonstration will require a more detailed consideration of what mechanical information might exist that could specify shape and be detected when an object is wielded.

\section{EXPERIMENT 3}

Our guiding hypothesis is that the pattern of resistances summarized in the inertia tensor (or more technically, the tissue consequences of that pattern) is determined by aspects of the object's shape and could, therefore, be in-

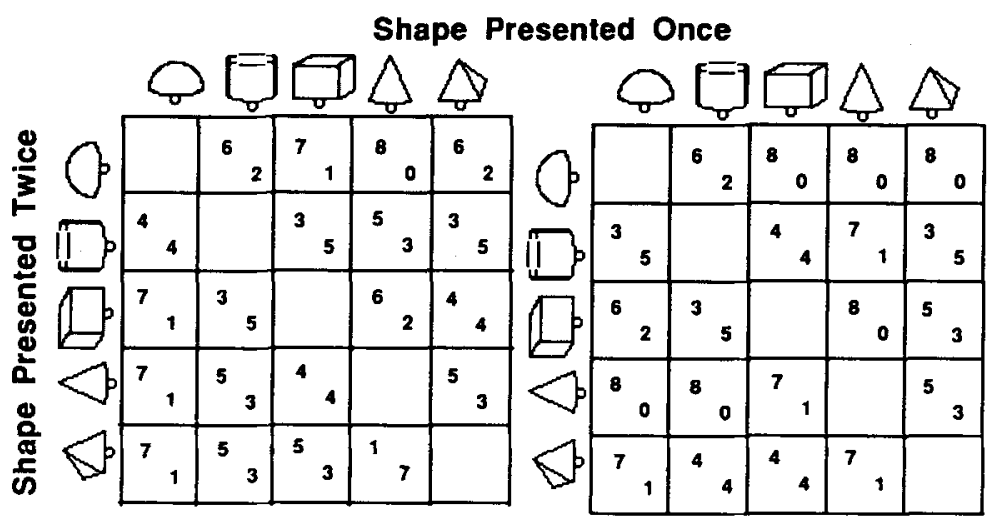

Figure 3. Results for the two sizes employed in Experiment 2. The illustration at the top of each column indicates which shape was presented once in a given comparison; the illustration for each row indicates which shape was represented twice in that comparison. The number in the upper left of each cell is the number of subjects who correctly picked the two objects of similar shape when presented with this combination; the number in the lower right indicates the number of errors. 
formative about the shape. An object that greatly resists being whipped but hardly resists being twisted is a long rod. Whereas perceiving the length of a rod only requires the detection of (the tissue deformation consequences of) $I_{x x}$, perceiving that it is a rod requires the detection of a higher order property comprising both $I_{x x}$ and $I_{z z}$ (or possibly, all three principal moments, two of which are identical in radially symmetrical objects).

Classical mechanics provides at least two properties that are of higher order in the sense that they are invariant over change of coordinates-namely, the trace, which is the sum of the three principal moments, and the determinant, which is the product (see, e.g., Bradbury, 1968). Neither the trace of an object's moments of inertia, nor the determinant, can be specific to shape, however, since they both vary with object size. What is needed is a quantity that reflects the spatial distribution of mass indifferent to size. Only a ratio of the principal moments could meet this criterion, and the ratio that makes immediate sense is that of the greatest principal moment to the least principal moment. The symmetries of the objects in the present series of experiments dictate that two of the principal moments of inertia are identical; accordingly, this particular ratio fully captures their distributional character. We will refer to this greatest/least ratio as an object's inertial index, and we note that it is comparable to the compactness measure applied to visual shape perception (Hildreth \& Ullman, 1989). Functionally, the inertial index provides a measure of the resistance to being rotationally accelerated around one axis relative to the resistance to being rotationally accelerated around another, orthogonal axis.

As a first step in the assessment of the inertial index's contribution to shape perception, this index was computed for each of the five shapes at both sizes. The results are given in Table 3. (Formulas for the moments of inertia of a hemisphere, parallelepiped, cylinder, cone, and pyramid can be found in textbooks of mechanics. ${ }^{3}$ The values in Table 3 also reflect the fact that the objects are being wielded at a certain distance from the center of mass, that distance being equal to the length of the handle plus the distance from the base of the object to its center of mass. The moment of inertia of the handles themselves is discounted.) Note that this inertial index does not reflect all distinctions that are salient visually; in particular, the difference between a square object and a round object of similar sizes is not captured in the object's inertia tensor. Note also that the indices differ across

Table 3

Inertial Indices for the Solids of the Three Sizes

\begin{tabular}{lrrr}
\hline \multicolumn{1}{c}{ Shape } & Size 1 & Size 2 & Size 3 \\
\hline Hemisphere & 1.45 & 1.45 & 1.45 \\
Cylinder & 2.12 & 2.53 & 2.66 \\
Parallelepiped & 2.00 & 2.19 & 2.15 \\
Cone & 11.16 & 14.66 & 14.97 \\
Pyramid & 9.87 & 11.66 & 11.70 \\
\hline
\end{tabular}

the sizes, because of slight differences in the proportions and also reflecting the fact that the objects were not wielded at their centers of mass.

If the perception of object shape by means of active touch involves the detection of inertial indices, then the distinctiveness of two solids should be related to the difference between their corresponding indices. This supposition holds well at the extremes. The index for the small cone has a magnitude 7.7 times greater than that for the small hemisphere, whereas the index for the small cylinder is only 1.1 times greater than the index for the parallelepiped. Correspondingly, the results of Experiments 1 and 2 indicate that the combination of hemispheres and cones was judged with ease, whereas the combination of parallelepipeds and cylinders was hardest to distinguish.

Within these two extremes, is the ratio of inertial indices (hereinafter abbreviated RII) a good predictor of the distinctiveness of two objects? A regression was performed on the results of Experiment 2, in which the number of confusions for each shape combination was rendered as a function of ratio of inertial indices. The dependency was significant $\left(R^{2}=.23, p<.05\right)$; however, a regression against the ratio of object masses showed a higher dependency $\left(R^{2}=.57, p<.001\right)$. Stepwise regression with both mass ratio and $R I I$ entered mass ratio first, yielding an overall $R^{2}$ of $.70(p<.001)$. The implication is that the translational inertia (mass) differences between objects constrained the judgments of subjects in Experiment 2 more than did the rotational inertia differences. This bias may have been conditioned by the nature of the task in Experiment 2, which encouraged subjects to find any basis for deciding on the sameness or difference among simultaneously and successively wielded objects. To circumvent this bias and to obtain, thereby, a more direct evaluation of RII, we turned to a task in which subjects had to identify (by name or by pointing) the shape of a singly wielded object. Because this identification task was expected to be more difficult than the comparison tasks, subjects were screened from a larger pool and given practice trials as in Experiment 1.

\section{Method}

Subjects. In the screening experiment, 24 undergraduates from the University of Connecticut served as subjects as partial fulfillment of class requirements. In addition, the subjects were informed of the possibility of participation in an upcoming experiment for payment. Thirteen subjects were male; 2 male and 1 female subject were left-handed. Of these subjects, 10 who reached a criterion on a comparison task were selected for the identification phase. Six of these subjects were male, and all were right-handed. The subjects in the second phase participated for a $\$ 5$ fee.

Materials. The objects of Size 1 were used in the screening phase; Sizes 1 and 2 were employed in the identification phase. The other materials were the same. In addition, for the identification phase, a display was used that consisted of the Size 3 objects; these solids were placed in a two-tiered platform such that the handles were not visible.

Apparatus. A different room was used, equal in size to that employed in Experiment 2; the room contained some equipment un- 
related to the current experiment. The display was positioned at a distance of about $3 \mathrm{~m}$ from the subject's chair.

Procedure. The screening task followed the same basic procedure as that in Experiment 2. Each subject was given 10 practice trials, comprising five in which they wielded two objects of the same shape, and four in the style of Experiment 2 (that is, in which subjects were presented two solids of one shape and one of a different shape); the 10th trial was one in which the shapes differed and was mixed in with the first 5 trials in order to provide more contrast. Each subject was given 10 main trials, in which each possible shape contrast was given once. It was decided randomly which shape in the comparison would be the different object; each subject was paired with another subject, who received the opposite combinations (i.e., if 1 subject received hemisphere-parallelepiped-parallelepiped and pyramid-cone-cone, there would be another subject who faced parallelepiped-hemisphere-hemisphere and cone-pyramid-pyramid).

Ten subjects who correctly identified the different shape on at least 7 of the 10 trials were recruited for the identification phase. For the main trials, objects were placed one at a time into one of the fixtures of the stand (the particular fixture was chosen according to the subject's preference). As depicted in Figure 4, the subject picked up the object and shook it behind a screen for as long as desired, and then identified the shape of the object, either by name (e.g., "pyramid" or "hemisphere"), or by indicating where on the platform its larger correspondent was situated (e.g., "upper middle" or "lower right"). In practice, the subjects most often identified the shape by name. Each of the 10 objects ( 5 shapes $\times 2$ sizes) was presented three times, and all $\mathbf{3 0}$ trials were randomized. In addition, there were 10 randomized practice trials (one with each object).

Five of the subjects were given feedback throughout the experiment; the remaining 5 received feedback only during practice. Feedback consisted of the experimenter's taking the object from the subject's hand and holding it above the screens. Before the main trials commenced, the experimenter took pains to prepare the subjects for the possibility that their performance on the current task might not be as high as in the previous experiment.

\section{Results and Discussion}

Figure 5 shows the number of confusions for each shape combination. The results resemble those of the previous experiments, although it should be kept in mind that a confusion in this experiment implies a case when an object

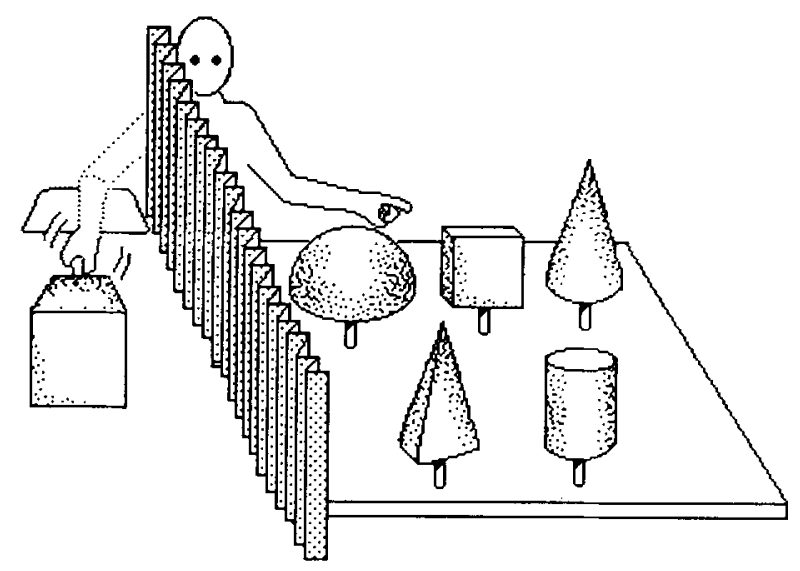

Figure 4. The depiction of the arrangement for Experiment 3 and, later, Experiment 4 (but not Experiments 1 and 2). A subject wielded a given object in the right hand, which was hidden by a screen, and indieated from a visible display of similar (but differently sized) objects which shape was being wielded.

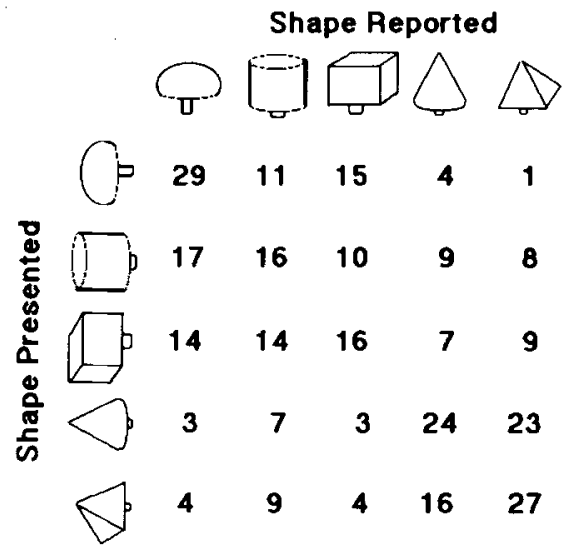

Figure 5. Results for the 10 subjects in Experiment 3. The illustration at the left of each row depicts the shape presented to the subject; the thustration at the top of each column represents the shape reported by the subject. Thus, a cone was identified as a cone 24 times, and a cone was identified as a cylinder 7 times.

of one shape was identified as having a second shape, whereas in Experiment 2, a confusion indicated a case for which a subject could not pick the different shape from a set comprising two examples of one shape and one of the other. According to analysis of variance, neither the variable of object size $[F(1,8)=3.14, p>.05]$ or of feedback versus no feedback $[F(1,8)=1.24, p>.05]$ had a significant effect on the number of correct identifications; the shape of the presented object was a significant factor $[F(4,32)=3.06, p<.05]$. Across all conditions, the mean number of correct identifications (11.3 out of 30 ) exceeded the level expected by chance (that is, 1 out of 5) according to $t$ test $[t(9)=4.59, p<.01] .^{4}$

These analyses reveal that the number of correct answers exceeded chance level. The next step was to consider any possible regularity in the incorrect answers. The number of confusions for each shape combination was regressed against RII for that combination. As before, mass ratio was included in a stepwise regression, but this failed to account for variance. The results of a regression against RII are shown in Figure 6. The regression indicates that RII is a good predictor of the confusability of a particular combination.

To test further the predictive value of RII, the number of confusions for the screening phase of this experiment were regressed against RII as well as mass ratio. Stepwise regression entered RII first, but also the ratio of masses, indicating some influence of the mass differences (as we might expect, since the screening task involved comparison in the manner of Experiment 2). The results of a regression against $\mathrm{RI}$ are given in Figure 7; the maximum number of confusions is now 24 , and yet the fit is still good for every combination except that of the parallelepiped and cylinder, which were confused less often than the ratio would predict. The fact that the task in the screening test was different from that in the main experiment confirms that the distinctiveness of objects of differ- 
ent shapes, however that distinctiveness is tested, depends on the distinctiveness of the inertial indices.

\section{EXPERIMENT 4}

Experiment 3 confirmed that RII is a good predictor of which shapes will be confused. To test the limits of this dependency, a version of the identification task was conducted in which subjects wielded shapes of more distinct sizes, Sizes 1 and 3 from Tables 1 and 2. Moreover, the subjects were not screened on the basis of a different task, and were thus naive as to the task, apart from the practice trials conducted at the same session.

\section{Method}

Subjects. Ten male undergraduates took part in Experiment 4 as partial fulfillment of a course requirement. Males were recruited because of the large size of some of the solids. All 10 subjects were right-handed, although they were not recruited on this basis.

Materials. The stand and screen arrangement from the preceding experiment was again employed. The solids of the middle size were substituted with a third group of solids, again of the same five shapes and again designed to have the same volume; the dimensions of these objects are given in the third column of Table 1, and the masses are reported in the third column of Table 2. The Size 2 objects were now placed into the display from Experiment 3, so that as before, the displayed objects were of a size not wielded by the subjects.

Apparatus. The experiment took place in a larger room than did the previous two experiments; this room contained some equipment unrelated to Experiment 4.

Procedure. The procedure from the identification phase of Experiment 3 was again employed. However, no feedback was pro-

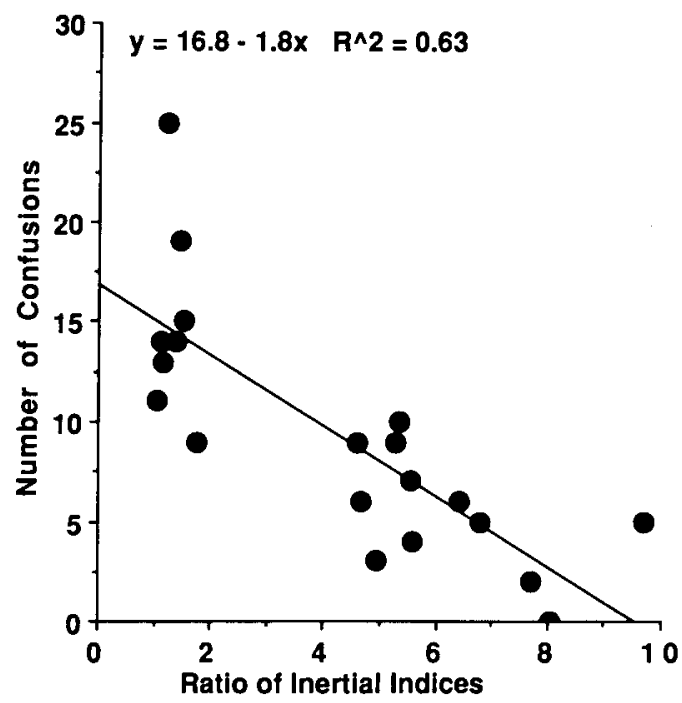

Figure 6. Regression of the number of confusions for each shape combination on the ratio of the inertial indices for the two shapes, for Experiment 3. There were 10 subjects, each of whom was presented with each shape three times at two sizes. Thus, 60 is the maximum number of confusions. For example, each of 10 subjects was presented with three Size 1 cones that could have been identified as cylinders, and three Size 1 cylinders that could have been identified as cones.

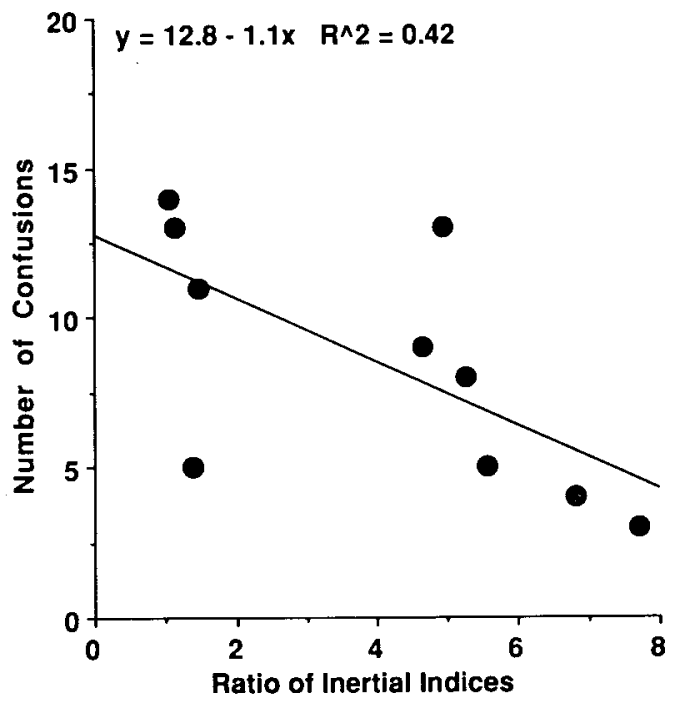

Figure 7. Regression of the number of confusions for each shape combination on the ratio of the inertial indices for the two shapes, for the $\mathbf{2 4}$ subjects in the screening portion of Experiment 3 . In the screening portion, a confusion was recorded if the subjects chose the wrong solid as different out of a group of three (as in Experiment 2). The maximum number of confusions was 24.

vided in the data-collection stage of the experiment, inasmuch as the provision of feedback did not significantly influence the results in Experiment 3.

\section{Results and Discussion}

Figure 8 shows the number of identifications for each shape. The average number of correct identifications was significantly greater than chance $[t(9)=2.46, p<.05]$. As in Experiment 3, the number of correct responses did not differ significantly according to object size $[F(1,9)<1, p>.05]$; object shape was also nonsignificant $[F(4,36)<1, p>.05]$, in contrast to the results of Experiment 3.

The number of confusions for each shape combination were regressed against RII, as well as the ratio of masses. Under stepwise regression, the ratio of masses did not enter as a factor. Figure 9 shows the results of the regression against RII, which yielded an $R^{2}$ of .70 . The results reveal that RII is also a good predictor of the confusability of two shapes for the sizes employed in Experiment 4.

\section{GENERAL DISCUSSION}

Dynamic touch, in the sense detailed by J. J. Gibson (1966), makes information available about mechanical properties of held or contacted objects. When an object is wielded, strains are induced on the tissues of the wielder (e.g., the wielder's hand). These tissue strains (and/or rates of strain) are structured, and this structure is determined by and specific to certain inertial properties of the object wielded. This specific, structured array of strains 


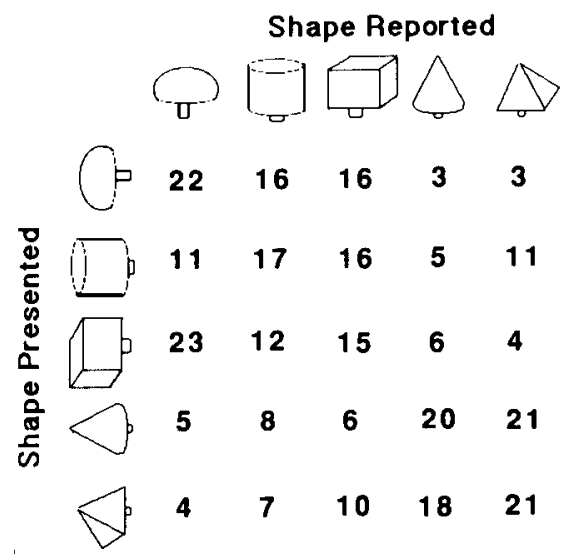

Figure 8. Results for the ten subjects in Experiment 4. The illustration at the left of each row depicts the shape presented to the subject; the illustration at the top of each column represents the shape reported by the subject. Thus, a cone was identified as a cone $\mathbf{2 0}$ times, and a cone was identified as a cylinder 8 times. The maximum number of identifications in each cell is 60 (10 subjects $\times 2$ sizes $\times 2$ repetitions).

or rates of strain (different magnitudes of strains or strain velocities in different directions) constitutes the information available to the perceiver. Just as the inertial properties of the environmental object can be summarized in the inertia tensor, a quantification of different resistances to rotational acceleration in different directions, the tissue deformation consequences determined by them are embodied in the strain tensor and/or rate of strain tensor, a quantification of different strains and/or rates of strain in different directions (Solomon \& Turvey, 1988; Turvey, Solomon, \& Burton, 1989). These lawfully generated arrays of strains and strain velocities might fruitfully be

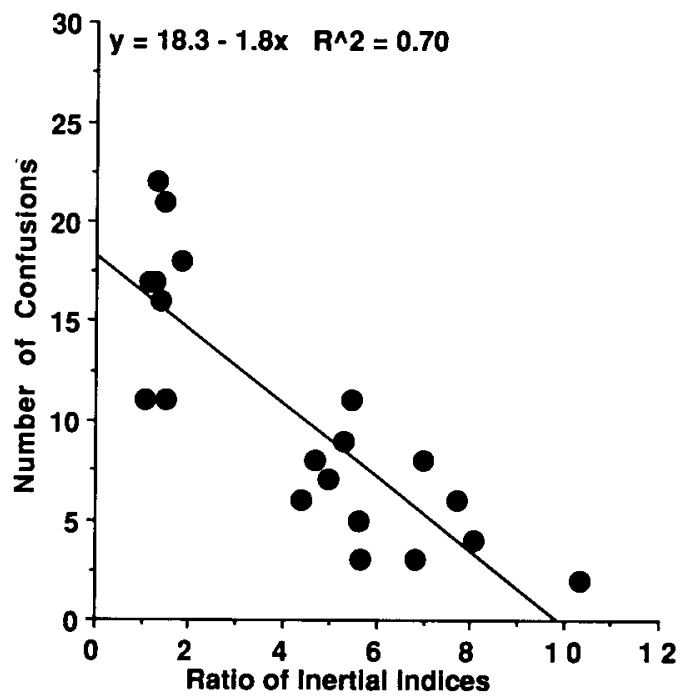

Figure 9. Regression of the number of confusions for each shape combination on the ratio of the inertial indices for the two shapes, for Experiment 4 . There were 10 subjects, each of whom was presented with each shape three times. likened to the optic array and optic flow field, respectively, discussed by J. J. Gibson (1979).

Mathematically, although there is a one-to-one mapping between object dimensions and inertial indices, the mapping from any particular set of inertial indices to the shape of the solid, as previously discussed, is one to many. This situation is found for most mechanical properties of solid objects. Nevertheless, the results of the comparison tasks and identification tasks reported here demonstrate that aspects of the shape of wielded objects can be perceived through dynamic touch-the perceptual ability of the subjects in these experiments was certainly less ambiguous than expected from the mathematical mapping, as alluded to in the introduction.

Unlike the visual case or the case of perception of shape by cutaneous and haptic touch (J. J. Gibson, 1966), this ability depends on detection of the tissue deformation consequences of mechanical properties of the object. The resistance of the object to rotational acceleration around orthogonal axes (or specifically, the tissue strains and/or rates of strain caused by this resistance) seems to be the detectable property, and its measure, the inertial index, is seen as a good predictor of the probability that an object of a particular shape will be identified as having a different particular shape. These mechanical implications of shape transcend the size of the object; subjects in these experiments had some success with three different sizes of solids.

Because the perception of shape through dynamic touching has little relation to geometric properties such as the number or orientation of edges, faces or vertices, it is not surprising that some properties salient to vision and to cutaneous and haptic touch are not perceivable through dynamic touching (at least under the paradigm used here). Most subjects reported being unable to tell if a wielded object was round or square; and indeed, there is no property within the inertia tensor to specify this distinction. A few subjects did demonstrate forms of shaking the solids that apparently revealed to them whether the object was round or square; informally, this method worked for the subjects who discovered it themselves, but it was not effective when suggested to other subjects.

The results of these experiments confirm that the perception of shape by means of dynamic touching does not necessarily reduce to the perception of surface shape, which is a reduction commonly assumed for the case of perception by means of cutaneous and haptic touching, and with vision. Subjects were able to detect a pervasive property of object shape, as opposed to properties localized at salient vertices, edges, and faces. This ratio of inertial indices is an aspect of object shape that can be detected through a course of exploration; inherently, it involves exploration along different axes of rotation. In a sense, the ratio of resistances around orthogonal axes is an invariant of object shape that emerges from a series of haptic transformations (strain transformations at the hand and wrist).

Consideration of this sort of detectable, pervasive invariant is inspired by J. J. Gibson's developing analysis 
(1951, 1973, 1979) of the properties detected when shape is visually perceived. It has long been recognized that objects will appear to have different shapes according to the perspective from which they are viewed, and the detection of a single shape from this family of perspective transformations has long been deemed a puzzle (Austin, 1962). J. J. Gibson (1979) argued that it was these very transformations that supported the perception of shape, in that invariant properties could be distilled from a series of varying transformations in a way that would be unavailable from a single, static viewpoint. Or, to quote a related sentiment from an earlier paper, "this ... radical hypothesis ... suggests that the perception of an object does not depend on a series of percepts, each of one image of the object, that is, perceptions of its forms and perspectives, but depends instead on the invariant features of the forms and perspectives over time ... and these invariants are 'formless' that is to say, they are not themselves forms"' (J. J. Gibson, 1973, p. 285).

Computationally minded researchers have also recognized the need for a characterization of shape that does not depend on surfaces; Marr (1982) provides a careful exemplar. Marr saw the problem of shape perception as a problem of finding the intrinsic coordinate frames in which aspects of the object could be described. He distinguished between vantage-centered coordinate systems, which yield distinct shapes for distinct viewpoints, and object-centered coordinate systems, which are by definition specific to the object considered. (An additional affinity of Marr's analysis in the visual case with the approach sketched in this report is Marr's emphasis on volumetric shape primitives as an alternative to surfacebased shape primitives. The former class of primitives are less simple but contain distribution information.)

In light of the preceding discussion, it is possible that there are certain shape differences that would be more distinctive under the explorations of dynamic touching than under forms of explorations that focus on the object surface. Consider a series of objects with similar surface shapes (e.g., a set of parallelepipeds) that differ according to how tall they are relative to the width of their bases. Such a distinction would be specified in the inertial index, but might be difficult to perceive visually or cutaneously, depending on how slight the differences are. Experiments are currently being designed that incorporate a set of objects like this. An added advantage of this kind of experiment is that the subject could give a metrical report as to the perceived dimensions of the object, by drawing a box with a ratio of height to base matching that of the wielded solid, for example. We plan to regress the reports of subjects against the inertial index of each solid to determine if the dependency continues to hold.

It may also be important to consider how the ability to perceive shape by dynamic touching develops. The ability to perceive is a skill, and a skill that develops (E. J. Gibson \& J. J. Gibson, 1955). One attitude toward this development, a perspective that can be attributed to the tradition of Berkeley, is that the same input (in the form of sensa- tions) is detected throughout the process, but that meaning is assigned to that input as the organism gains experience. A second tradition, associated with the Gibsons, points out that the claim that young organisms can detect the same information as adults is no more plausible, a priori, than the claim that young organisms can perform the same physical movements as adults. This tradition asserts that animals do learn to perceive and do learn to perceive better, and that the assignment of meaning to this information is not the source of this ability. A recent example of this approach was provided by Epstein, Hughes, Schneider, and Bach-y-Rita (1989) for vibrotactile displays. The authors demonstrated that some subjects did improve in their ability to distinguish vibrotactile patterns with practice, but that the grouping of these patterns remained relatively constant. Thus, subjects could improve in their ability to detect the same information. A similar paradigm could be used to assess whether subjects can improve in their ability to perceive shape, and whether the inertial index still predicts their confusions even when the number of confusions may be declining.

\section{REFERENCES}

Austin, J. L. (1962). Sense and sensibilia. Oxford, U.K.: Oxford University Press.

Bingham, G. P. (1987). Kinematic form and scaling: Further investigations on the visual perception of lifted weight. Joumal of Experimental Psychology: Human Perception \& Performance, 13, 155-177.

Bradbury, T. C. (1968). Theoretical mechanics. New York: Wiley.

Brodie, E. E. (1988). Sex and hand-preference effects in the simultaneous and consecutive discrimination of lifted weight. Perception \& Psychophysics, 43, 326-330.

Carlin, B. P., Ge Grand, A. E. (in press). A sample reuse method for accurate parametric empirical Bayes confidence intervals. Journal of the Royal Statistical Society.

Epstein, W., Hughes, B., Schneider, S. L., \& Bach-y-Rita, P. (1989). Perceptual learning of spatiotemporal events: Evidence from an unfamiliar modality. Journal of Experimental Psychology: Human Perception \& Performance, 15, 28-44.

GIBSON, E. J., \& GiBSON, J. J. (1955). Perceptual learning: Differentiation or enrichment? Psychological Review, 62, 32-41.

Gibson, J. J. (1951). What is a form? Psychological Review, 58, 403412. Reprinted in E. Reed \& R. Jones (Eds.), Reasons for realism (pp. 303-314). Hillsdale, NJ: Erlbaum.

Gibson, J. J. (1963). The useful dimensions of sensitivity. American Psychologist, 18, 1-15. (Reprinted in E. Reed \& R. Jones (Eds.), Reasons for realism, 1982, pp. 350-373. Hillsdale, NJ: Erlbaum.)

GIBson, J. J. (1966). The senses considered as perceptual systems. Boston: Houghton-Mifflin.

GiBson, J. J. (1973). On the concept of "formless invariants" in visual perception. Leonardo, 6, 43-45. (Reprinted in E. Reed \& R. Jones (Eds.), Reasons for realism, 1982, pp. 284-288. Hillsdale, NJ: Erlbaum.)

GiBson, J. J. (1979). The ecological approach to visual perception. Hillsdale, NJ: Erlbaum.

Gilden, D. L., \& ProffitT, D. R. (1989). Understanding collision dynamics. Journal of Experimental Psychology: Human Perception \& Performance, 15, 372-383.

GoldsteIn, H. (1980). Classical mechanics. Reading, MA: AddisonWesley.

GUIARD, Y. (1987). Asymmetric division of labor in human skilled bimanual action: The kinematic chain as a model. Journal of Motor Behavior, 19, 486-517.

Hildreth, E., \& Ullman, S. (1989). The computational study of vi- 
sion. In M. E. Posner, (Ed.), Foundations of cognitive science (pp. 581-630). Cambridge, MA: Bradford Press.

JENKINS, W. O. (1947). The tactual discrimination of shapes for coding aincraft-type controls. In P. M. Fitts, (Ed.), Psychological research in equipment design (pp. 199-205). Washington, DC: U.S. Govemment Printing Office.

KAC, M. (1966). Can one hear the shape of a drum? Mathematics Monthly, 13, 21-23.

KiBgle, T, W. B. (1985), Classical mechanics. London: Longman.

KlatzKY, R. L., Lederman, S. J., \& MetzGer, V. A. (1985). Identifying objects by touch: An "expert system." Perception \& Psychophysics, 37, 299-302.

LederMaN, S. J., \& KLATZKY, R. L. (1987). Hand movements: A window into haptic object recognition. Cognitive Psychology, 19, 342-368.

LOOMns, J., \& LedermaN, S. J. (1986). Tactual perception. In K. R. Boff, L. Kaufman, \& J. P. Thomas, (Eds.), Handbook of perception and human performance: Vol. II. Cognitive processes and performance. New York: Wiley.

Marascuilo, L. A. (1970). Extensions of the significance test for oneparameter signal detection hypotheses. Psychometrika, 35, 237-243.

MARR, D. (1982). Vision. San Francisco: W. H. Freeman.

Proffitt, D. R., GILDEN, D. L. (1989). Understanding natural dynamics. Joumal of Experimental Psychology: Human Perception \& Performance, 15, 384-393.

Roland, P. E., Mortensen, E. (1987). Somatosensory detection of microgeometry, macrogeometry, and kinesthesia in man. Brain Research Reviews, 12, 1-42.

Runzson, S., Frykholm, G. (1981). Visual perception of lifted weight. Journal of Experimental Psychology: Human Perception \& Performance, 8, 733-740.

Solomon, Y., TuRveY, M. T. (1988). Haptically perceiving the distance reachable with hand-held objects. Joumal of Experimental Psychology: Human Perception \& Performance, 14, 404-427.

Solomon, H. Y., TURveY, M. T., \& Burton, G. (1989a). Gravitational and muscular variables in perceiving extent by wielding. Ecological Psychology, 1, 265-300.
Solomon, H. Y., Turvey, M. T., \& Burton, G. (1989b). Perceiving rod extents by wielding: Haptic diagonalization and decomposition of the inertia tensor. Joumal of Experimental Psychology: Human Per. ception \& Performance, 15, 58-68.

Strang, G. (1986). Introduction to applied mathematics. Wellesley, MA: Wellesley-Cambridge Press.

Strerı, A., PÊcheux, M.-G. (1986). Tactual habituation and dis. crimination of form in infancy: A comparison with vision. Child Development, 57, 100-104.

Turvey, M. T., Solomon, H. Y., Burton, G. (1989). An ecological analysis of knowing by wielding. Joumal of the Experimental Analysis of Behavior, 52, 387-407.

Yavorsky, B. \& Detlaf, A. (1972). Handbook of physics (Nicholas Weinstein, Trans.). Moscow: Mir.

\section{NOTES}

1. This is not to imply that mechanical properties of objects are not visually perceivable - in fact, the rich literature of the "kinematics specifies dynamics" principle (e.g., Bingham, 1987; Runeson and Frykholm, 1981) centers on this ability. But see also Gilden and Proffitt (1989) and Proffitt and Gilden (1989).

2. If the method of Marascuilo (1970) is employed to assess the significance of the $d^{\prime}$ statistics associated with each subject, 3 of the subjects show $d^{\prime}$ significant at $p<.01$, and an additional 2 show $d^{\prime}$ significant at $p<.05$.

3. One of the most comprehensive listings is in Yavorsky and Detlaf (1972, pp. 88-90).

4. Carlin and Gelfand (in press) explore an alternate approach to determining the level expected by chance, using some of these data.

(Manuscript received September 5, 1989; revision accepted for publication June 25,1990 .) 